\title{
Análise do Impacto do Programa Saúde na Escola sobre a Violência e o Consumo de Substâncias llíitas dos Jovens nas Escolas Brasileiras
}

\section{The Impact Analysis of the School Health Program on Violence and Illicit Substance Use by Young People in Brazilian Schools}

\author{
Kalinca Léia Becker ${ }^{\mathrm{a}}$
}

\begin{abstract}
Resumo: O estudo analisa o impacto do Programa Saúde na Escola (PSE) na prevenção da violência e no uso de álcool, tabaco e outras drogas. Com base nas informações da Pesquisa Nacional de Saúde do Escolar, Pense 2015, estima-se o efeito médio do tratamento (escolas que aderiram ao PSE) através do propensity score matching e do método de Lewbel (2012), que se baseia na construção de instrumentos internamente para lidar com o possível viés das características não observáveis que influenciam o tratamento. Também é estimado o efeito do tratamento quantílico. Os resultados indicam que o PSE contribui para a prevenção do consumo de drogas, cigarros e de brigas. No caso do álcool e do bullying, não foram observados resultados significativos.
\end{abstract}

Palavras-chave: Programa Saúde na Escola. Violência. Drogas.

\begin{abstract}
The study analyzes the impact of School Health Program (PSE) on the prevention of violence and on the consumption of alcohol, tobacco and other drugs. Based on National Student Health Survey, Pense 2015, we estimated the average treatment effect (schools that adhered the PSE) through propensity score matching and the Lewbel method (2012), which is based on the construction of instruments internally to deal with the possible bias of the unobserved characteristics that influence the treatment. The quantile treatment effects was also estimated. The results indicated that the PSE contributes to the prevention of drug and cigarette consumption and fights. In the case of alcohol and bullying, no significant results were observed.
\end{abstract}

Keywords: School Health Program. Violence. Drugs.

JEL Classification: I18; K42; C21.

\footnotetext{
a Universidade Federal de Santa Maria, Departamento de Economia e Relações Internacionais. Rio Grande do Sul, Santa Maria, Brasil.
} 


\section{1 lntrodução}

A violência e o consumo de drogas tornaram-se problemas recorrentes nas escolas brasileiras e estão entre os tantos desafios a serem enfrentados pelos profissionais e gestores da educação. Conforme Becker e Kassouf (2016), das escolas observadas na Prova Brasil de 2009, 47\% registraram uma agressão física de um aluno. O VI Levantamento nacional sobre o consumo de drogas psicotrópicas entre estudantes do ensino fundamental e médio das redes pública e privada de ensino nas 27 capitais brasileiras 2010 (BRASIL, 2010) identificou que 25,5\% dos estudantes do ensino básico já experimentaram alguma droga ilícita ao longo da vida.

Essas situações prejudicam o aprendizado dos alunos e, consequentemente, o processo de formação do capital humano. Vários estudos na literatura nacional apresentam evidências de que a violência na escola reduz a proficiência do aluno (GAMA; SCORZAFAVE, 2013; TEIXEIRA; KASSOUF, 2015; FIRPO; JALES; PINTO, 2015; MONTEIRO; ROCHA, 2017). Já o consumo substâncias psicotrópicas pode comprometer de forma permanente a capacidade cognitiva dos jovens. Almeida e Araújo Júnior (2016) apresentam evidências de que a exposição aos fatores de risco, como cigarro e álcool, se relaciona de forma direta com o atraso escolar. Além disso, tal consumo pode desencadear outros problemas de saúde, como dependência química, comprometimento cardiovascular, respiratório entre outros, aumentando a demanda pelo sistema público de saúde, o que representa maiores gastos para a sociedade (BECKER, 2017b).

Diante disso, entre as linhas de ação do Programa Saúde na Escola (PSE) estão a prevenção ao uso do álcool, tabaco e outras drogas e a promoção da cultura da paz e prevenção das violências. Esse programa foi instituído pelo Decreto $\mathrm{n}^{\mathrm{O}}$ 6.286, de 5 de dezembro de 2007, com o objetivo fortalecer ações para o enfrentamento das vulnerabilidades que comprometem o pleno desenvolvimento dos alunos. Todas as escolas públicas estão aptas a receber os recursos para desenvolver as atividades previstas nas linhas de ação do PSE, desde que o gestor municipal tenha realizado o cadastro no sistema eletrônico do programa e tenha solicitado recursos para a escola. Assim, se espera que as ações educativas e participativas articuladas nas escolas que aderiram ao PSE possam criar mecanismos de valorização da cultura da paz e ampliação do zelo com a saúde (BRASIL, 2011a).

Por se tratar de um programa que demanda a alocação de recursos públicos e cujos resultados esperados são de grande relevância social, destaca-se a importância de se buscar evidências empíricas do impacto do PSE na prevenção da violência e do consumo de substâncias ilícitas entre jovens.

Este estudo pretende contribuir nesse sentido estimando o efeito do tratamento (escolas que aderiram ao PSE) por meio de cinco resultados: a proporção de alunos na escola que declaram já ter consumido drogas, álcool e cigarros, en- 
volvimento em brigas e prática de bullying. Para isso, estima-se o efeito médio do tratamento nos tratados (ATT) através dos métodos de propensity score matching, que se baseia nas características observáveis dos alunos e das escolas, e do método de Lewbel (2012), que busca controlar o possível viés causado pela influência das características não observáveis sobre o tratamento, através da construção de instrumentos internamente a partir da heterocedasticidade do termo de erro.

Além disso, é possível que o efeito do tratamento seja diferente ao longo da distribuição das variáveis de resultados. Por exemplo, é possível que uma política para a prevenção do uso de cigarro produza resultados distintos nas escolas onde poucos alunos fumam em comparação às escolas nas quais muitos alunos fazem uso de tal substância. Por isso, também será estimado o efeito do tratamento quantílico.

Por fim, são estimadas bounds para o efeito de tratamento, conforme a metodologia proposta por Oster (2016), como estratégia adicional para se observar se as estimativas do efeito tratamento são viesadas em decorrências das características não observáveis.

\section{Revisão de Literatura}

Essa seção apresenta uma breve revisão dos estudos que buscam evidências empíricas do efeito dos programas realizados nas escolas para prevenir o consumo de drogas e a violência entre os alunos. Também contempla uma breve descrição do Programa Saúde na Escola.

\subsection{Evidências do Efeito dos Programas de Prevenção do Consumo de Drogas e Violência na Escola}

A escola tem um papel fundamental na formação das crianças e jovens, pois nesse espaço passam uma parte do seu tempo diário e, além de aprender conteúdos previstos na grade curricular, interagem com colegas e professores e podem obter informações sobre os malefícios do consumo de substâncias psicotrópicas ou da prática da violência.

Conforme Jacob e Lefgren (2003), a escola pode prevenir o comportamento disruptivo dos alunos através do ensino e do monitoramento. Essa relação é chamada de "efeito capacitação", que sugere que manter os jovens ocupados e fora das ruas pode diminuir o engajamento em atividades ilícitas. Porém, os autores acrescentam que, se o ambiente escolar for caracterizado com a presença da violência, o "efeito concentração" geográfica dos alunos nesse ambiente aumenta a probabilidade dos conflitos e pode levar ao engajamento em atividades ilícitas.

Através das variações nas leis de idade mínima permitida para o abandono escolar entre os estados dos Estados Unidos, Anderson (2014) examina a relação entre 
a evasão e as taxas de detenção juvenil. As evidências, obtidas através da estimação do método de diferenças em diferenças, sugerem que os requisitos mínimos de idade para o abandono escolar têm um efeito significativo e negativo sobre as taxas de crimes violentos ou contra a propriedade, para indivíduos de 16 a 18 anos. Conforme o autor, esses resultados são uma evidência do efeito capacitação da escola.

Ttofi e Farrington (2011) realizam uma revisão de literatura da eficácia dos programas antibullying nas escolas, analisando estudos que comparam os grupos de tratamento e de controle. Os autores observaram que, em geral, os programas realizados nas escolas diminuem o bullying entre $20 \%$ e $23 \%$ e a vitimização entre $17 \%$ e $20 \%$. Os programas mais eficazes foram os mais intensivos, que incluíam reuniões com os pais e métodos disciplinares rigorosos.

Espelage, Low, Polanin e Brown (2013) avaliam o efeito do Middle school program - second step: student success through prevention (SS-SSTP) sobre agressão, vitimização e violência sexual dos alunos do ensino médio através de um estudo de coorte longitudinal no qual foram aleatorizados alunos de 36 escolas americanas nos grupos de controle e tratamento. No grupo de tratamento, os professores implementaram lições semanais que incluíam empatia, comunicação, prevenção do bullying e habilidades para resolver conflitos. As análises multinível indicaram que os alunos nas escolas do grupo de tratamento apresentaram 42\% menor probabilidade de reportar uma agressão física em relação aos alunos nas escolas do grupo controle. Não foram observados efeitos significativos do SS-SSTP para agressão verbal, vitimização entre pares, provocações homofóbicas e violência sexual.

Faggiano et al. (2010) avaliam a eficácia de um programa de prevenção do abuso de substâncias psicotrópicas nas escolas através do EU-Dap (teste de prevenção de toxicodependência na área da droga), no qual estudantes de sete países europeus foram aleatorizados para participar da intervenção, que incluiu no currículo assuntos relacionados aos malefícios do uso dessas substâncias. Os resultados das estimações de modelos de regressão multinível, com informações coletadas antes da intervenção e 18 meses após, indicaram que o programa foi capaz de reduzir o consumo do álcool e da cannabis, porém não foram observados resultados significativos para o tabagismo.

Caria, Faggiano, Bellocco e Galanti (2011) avaliam o efeito desse mesmo programa especificamente sobre o consumo do álcool, analisando, separadamente, os estudantes que nunca haviam bebido ou bebiam esporadicamente no momento de implementação do programa e aqueles que bebiam com frequência. Para os primeiros, os resultados indicaram que os estudantes do grupo tratamento apresentaram menor probabilidade de tornarem-se consumidores frequentes em comparação ao grupo controle. Já para os estudantes que bebiam com frequência, os resultados do programa não foram significativos. 


\subsection{O Programa Saúde na Escola (PSE)}

O Programa Saúde na Escola (PSE), instituído pelo Decreto nº 6.286 de 5 de dezembro de 2007 e desenvolvido intersetorialmente pelos Ministérios da Saúde e da Educação, tem como objetivo contribuir para o fortalecimento de ações na perspectiva do desenvolvimento integral e proporcionar à comunidade escolar a participação em programas e projetos que articulem saúde e educação para o enfrentamento das vulnerabilidades que comprometem o pleno desenvolvimento de crianças, adolescentes e jovens brasileiros (BRASIL, 2011a). Embora o PSE tenha sido instituído em dezembro de 2007, a primeira portaria que habilitou os municípios a receber o repasse financeiro para o PSE é de 20 de dezembro de 2011, Portaria $\mathrm{n}^{\circ} 3.014$.

O PSE trabalha em várias linhas de ação, e este estudo pretende analisar especificamente o impacto do programa em duas dessas linhas, que são a prevenção ao uso do álcool, tabaco e outras drogas e a promoção da cultura da paz e prevenção das violências. Essas ações consistem em realizar atividades no cotidiano da escola, abordando temáticas como bullying, discriminação, preconceito, riscos e danos do uso de álcool, tabaco, crack e outras drogas. Além das ações educativas e participativas (rodas de conversa, teatro, dinâmicas, narrativas e outras), o programa prevê o acolhimento e acompanhamento na rede de saúde dos educandos com danos decorrentes do uso abusivo e da dependência de substâncias psicotrópicas (BRASIL, 2011a).

Essas práticas, ao mesmo tempo que convocam a comunidade escolar a prevenir e enfrentar a violência, o consumo de drogas, álcool e cigarros, criam mecanismos de valorização da cultura da paz e ampliação do zelo com a saúde, que recolocam o sujeito no centro do paradigma do cuidado, compreendendo-o nas dimensões física, psicológica, social e cultural (BRASIL, 2011a). Assim, espera-se que as ações educativas e participativas articuladas nas escolas que aderiram ao PSE possam contribuir para a prevenção ao uso de álcool, tabaco e outras drogas e para a prevenção das violências.

A adesão ao programa é um processo de pactuação de compromissos firmados entre os secretários municipais de saúde e de educação com os ministérios da saúde e da educação. Conforme a Portaria Interministerial $n^{0}$ 1.413, de 10 de julho de 2013, que definiu as regras e critérios para adesão, todos os municípios estão aptos a aderir ao PSE, cadastrando-se no sistema eletrônico do programa. Nesse processo, o município deverá realizar a indicação das equipes de atenção básica, das escolas da rede pública, do número de educandos participantes e das ações a serem realizadas. 


\section{Metodologia}

O propósito deste estudo é estimar o efeito causal do PSE nas linhas de ação que visam a prevenção do uso do álcool, cigarro e outras drogas, bem como a promoção da cultura da paz e a prevenção das violências, com base nas informações da Pense 2015. Dessa forma, são analisados cinco resultados, que são a proporção de alunos na escola que declaram já ter consumido drogas, álcool e cigarros, se envolvido em brigas e praticado bullying.

Para isso, serão utilizados três métodos de estimação. O primeiro é o estimador por propensity score matching, que busca estimar o efeito médio do tratamento nos tratados $\left(\beta_{\text {ATT }}\right)$. Para isso, considera-se, primeiramente, a seguinte equação:

$$
\beta_{\text {ATT }}=E\left(Y_{i}^{1}-Y_{i}^{0}\right)
$$

em que $Y_{i}$ é o resultado observado da escola $i$ se houve adesão ao programa $\left(Y_{i}^{1}\right)$ e, no caso de não adesão, $\left(Y_{i}^{0}\right)$. Como, na prática, $Y_{i}^{1}$ e $Y_{i}^{0}$ não são observáveis para a mesma escola $i$, considera-se $Y^{1}$ como os resultados potenciais para o grupo de tratamento (escolas que aderiram ao PSE) e $Y^{0}$, como os resultados potenciais para o grupo controle (escolas que não aderiram ao PSE), respectivamente.

Dessa forma, para estimar $\beta$ é preciso encontrar indivíduos pertencentes aos grupos de tratamento e controle que possam ser comparados depois de ajustados às características observadas contidas em um vetor $X_{i}$ de características observáveis, com base na hipótese de independência condicional $Y^{1}, Y^{0}{ }_{\Perp} S_{i} \mid X_{i}$, na qual $S$ é o tratamento, ou seja, a adesão da escola ao PSE (ANGRIST; PISCHKE, 2009). Se o vetor $X_{i}$ contém as informações das escolas no grupo controle e de tratamento, é possível construir o contrafatual para a escola tratada e, assim se estimar o resultado da escola no caso de não adesão ao programa.

O propensity score ou probabilidade de a escola ser tratada, $p\left(X_{i}\right)$, permite encontrar o efeito do tratamento para cada valor de $X_{i}$ através da diferença das médias de $Y^{1}$ e $Y^{0}$ (ROSENBAUM; RUBIN, 1985). Dessa forma, a equação 1 pode ser reescrita como:

$$
\beta_{\text {ATT }}=\mathrm{E}\left\{\left[\mathrm{Y}_{\mathrm{i}} \mid \mathrm{p}\left(\mathrm{X}_{\mathrm{i}}\right), \mathrm{S}_{\mathrm{i}}=1\right]-\mathrm{E}\left[\mathrm{Y}_{\mathrm{i}} \mid \mathrm{p}\left(\mathrm{X}_{\mathrm{i}}\right), \mathrm{S}_{\mathrm{i}}=0\right]\right\}
$$

Estima-se o propensity score através de um modelo logit, fazendo o matching do grupo de tratamento com o grupo controle a partir da estimação por kernel (uma escola tratada comparada com uma ponderação das escolas do controle).

No entanto, o efeito médio pode não refletir completamente a influência do tratamento sobre os resultados potenciais, visto que os resultados podem ser diferentes ao longo da distribuição. Por isso, o segundo método a ser utilizado é o efeito do tratamento quantílico. Partindo-se da hipótese de variação exógena do 
tratamento nos resultados potenciais, a estimação pode ser feita pela regressão quantílica clássica, como sugerida por Koenker e Bassett (1978):

$$
\left(\hat{\beta}^{\tau}, \hat{\gamma}^{\tau}\right)=\arg \min _{\beta, \gamma} \sum W_{i} \times \rho_{\tau}\left(Y_{i}-X_{i} \gamma-S_{i} \beta\right)
$$

na qual $\rho_{\tau}(\mathrm{u})=\mathrm{u} \times\{\tau-1(\mathrm{u}<0)\}$ e $W_{i}$ se trata dos pesos. Tal estimador produz erros padrões analíticos, que são consistentes também na presença de heterocedasticidade.

Porém, a adesão ao PSE não é aleatória, conforme descrito no item 2.2 deste artigo. Um tratamento não é exógeno e a possibilidade de haver variáveis omitidas que influenciam o resultado podem levar a estimativas viesadas do efeito do tratamento, estimado por propensity score matching ou por regressões quantílicas.

Nesse caso, o ideal seria obter uma variável instrumental exógena que pudesse ser usada nas estimativas. Na falta dessa variável, muitos estudos têm utilizado o método de Lewbel (2012) para estimar o efeito causal (ERMAN; HOU, 2013; TIGRE; SAMPAIO; MENEZES, 2016). Embora essa técnica forneça estimativas menos confiáveis, o método tem produzido resultados semelhantes aos obtidos através de estimações com os instrumentos tradicionais.

Dessa forma, o terceiro método utilizado neste estudo baseia-se em Lewbel (2012), que parte da hipótese de que, se as correlações dos erros são causadas por fatores não observados, a identificação dos parâmetros estruturais pode ser obtida a partir de regressores não correlacionados com o produto dos erros heterocedásticos. Dessa forma, o método consiste em explorar a heterocedasticidade dos erros no primeiro estágio da regressão para gerar internamente instrumentos que possibilitem a identificação do sistema triangular de equações 4, 5 e 6. Se U é uma variável omitida, que afeta a variável endógena de tratamento $\mathrm{S}$ e, também, o resultado Y, é possível identificar o efeito causal de S em Y, denotado por $\beta$, através do método dos momentos generalizados (GMM):

$$
\begin{gathered}
Y=X^{\prime} \beta_{1}+\beta S+\varepsilon_{1} \\
S=X^{\prime} \beta_{2}+\varepsilon_{2} \\
E\left[X \varepsilon_{1}\right]=0, \quad E\left[X \varepsilon_{2}\right]=0, \operatorname{Cov}\left[Z, \varepsilon_{1} \varepsilon_{2}\right]=0
\end{gathered}
$$

nas quais $\varepsilon_{1}=\alpha_{1} U+V_{1}$ e $\varepsilon_{2}=\alpha_{2} U+V_{2}$, sendo $V_{1}$ e $V_{2}$ os erros idiossincráticos. $Z$ é um subconjunto de $X$, de modo que nenhuma informação de fora do modelo especificado é necessária.

A equação 6 apresenta as condições necessárias para a identificação e estimação, assim como a heterocedasticidade em $\varepsilon_{i}$. Quanto maior o grau da heterocedasticidade, maior será a correlação dos instrumentos gerados com as variáveis 
endógenas. A presença de heterocedasticidade pode ser analisada através do teste de Breusch-Pagan.

O teste J de Hansen de sobreidentificação das restrições de momento GMM também pode ser implementado para verificar a validade dos pressupostos do modelo, em especial da equação 6. A hipótese nula é de que os instrumentos são válidos, isto é, não correlacionados com o termo de erro e que os instrumentos excluídos assim são feitos de modo correto da equação estimada. A rejeição da hipótese nula põe em dúvida a validade dos instrumentos. Já o teste de Kleibergen-Paap Wald, de significância conjunta dos instrumentos no primeiro estágio da regressão, permite observar se os instrumentos são fracos, de modo que a rejeição da hipótese nula sugere que os instrumentos internos gerados são relevantes para o tratamento.

\subsection{Bounds para o Efeito de Tratamento}

Como estratégia adicional, utiliza-se o método proposto por Oster (2016) para estimar bounds para o efeito de tratamento, com base no pressuposto de que a seleção nos controles observáveis $X$ é proporcional à seleção nos controles não observados $U$. Dessa forma, pode-se definir um coeficiente de proporcionalidade $\delta$ para fornecer informações sobre como as não observáveis se relacionam com o tratamento $S$, dado que é conhecido como as observáveis se relacionam com tal tratamento. Dessa forma, $\delta \frac{\sigma_{X S}}{\sigma_{X}}=\frac{\sigma_{U S}}{\sigma_{U}}$,em que $\sigma_{\mathrm{XS}}=\operatorname{cov}(\mathrm{X}, \mathrm{S}), \sigma_{\mathrm{US}}=\operatorname{cov}(\mathrm{U}, \mathrm{S}), \sigma_{\mathrm{X}}=\operatorname{var}(\mathrm{X})$
e $\sigma_{\mathrm{U}}=\operatorname{var}(\mathrm{U})$.

As equações 7, 8 e 9 fornecem os $R$-quadrados definidos por $\mathrm{R}_{\max }, \mathrm{R}_{1}$ e $\mathrm{R}_{2}$, respectivamente. A equação 9 inclui um conjunto restrito de controles $M$, ortogonal com $X$ e $U$ :

$$
\begin{aligned}
& Y=\alpha+\beta S+X^{\prime} \gamma+U+\varepsilon_{\text {max }} \\
& Y=\alpha_{1}+\beta_{1} S+X^{\prime} \gamma_{1}+\varepsilon_{1} \\
& Y=\alpha_{2}+\beta_{2} S+M^{\prime} \gamma_{2}+\varepsilon_{2}
\end{aligned}
$$

A partir dessas informações, calcula-se $B(\delta)=\delta \frac{\left(\beta_{1}-\beta_{2}\right)\left(R_{\max }-R_{1}\right)}{R_{1}-R_{2}}$. Se as variáveis mais importantes para explicar o tratamento estiverem contidas no vetor $X$, a estimativa de $\delta$ deve ser maior que um. Para haver aleatoriedade nos movimentos da variável de resultado, Oster (2016) recomenda que sejam considerados valores para o $\mathrm{R}_{\max }$ variando de 0,7 até 1 . 


\section{Dados e Descrição das Variáveis}

Os dados são da Pesquisa Nacional de Saúde do Escolar (Pense), realizada em 2015 pelo Instituto Brasileiro de Geografia e Estatística (IBGE), com o objetivo de ampliar o conhecimento sobre a saúde dos adolescentes e subsidiar as políticas públicas relativas a esse grupo. A partir de uma amostra de escolas, foram coletadas informações de diversos fatores de risco à saúde, além das condições socioeconômicas dos estudantes do nono ano (oitava série) do ensino fundamental. Também foram coletadas informações básicas das escolas, fornecidas pelos diretores das unidades selecionadas. A escolha do nono ano do ensino fundamental teve como justificativa o mínimo de escolarização considerada necessária para responder ao questionário autoaplicável e também a proximidade da idade de referência preconizada pela Organização Mundial da Saúde (OMS), que é de 13 a 15 anos.

A Tabela 1 apresenta a descrição das cinco variáveis de resultados, que são a proporção de alunos na escola que declaram já ter consumido drogas, álcool e cigarros, se envolvido em brigas e praticado de bullying. A tabela também apresenta a média e o desvio padrão dos alunos que declaram já ter realizados tais atividades nas escolas que aderiram ao PSE e nas que não aderiram.

Tabela 1 - Definição das variáveis e estatísticas descritivas

\begin{tabular}{|c|c|c|c|}
\hline \multirow{2}{*}{\multicolumn{2}{|c|}{$\frac{\text { Descrição }}{\text { Variáveis de resultados }}$}} & \multicolumn{2}{|c|}{ MédiaI } \\
\hline & & PSE & Não PSE \\
\hline Droga & $\begin{array}{l}\text { Proporção de alunos na escola que responderam que uma } \\
\text { vez na vida já usaram alguma droga, tais como: maconha, } \\
\text { cocaína, crack, cola, "loló", lança-perfume, ecstasy, oxy, etc. }\end{array}$ & $\begin{array}{c}0.087 \\
(0.002)\end{array}$ & $\begin{array}{c}0.101 \\
(0.002)\end{array}$ \\
\hline Cigarro & $\begin{array}{l}\text { Proporção de alunos na escola que responderam que uma } \\
\text { vez na vida já fumaram cigarro, ainda que uma ou duas } \\
\text { tragadas. }\end{array}$ & $\begin{array}{c}0.193 \\
(0.002)\end{array}$ & $\begin{array}{c}0.215 \\
(0.003)\end{array}$ \\
\hline Álcool & $\begin{array}{l}\text { Proporção de alunos na escola que responderam que uma } \\
\text { vez na vida já tomaram uma dose de bebida alcoólica. }\end{array}$ & $\begin{array}{c}0.524 \\
(0.003)\end{array}$ & $\begin{array}{c}0.546 \\
(0.003)\end{array}$ \\
\hline Briga & $\begin{array}{l}\text { Proporção de alunos na escola que responderam que nos } \\
\text { últimos } 12 \text { meses se envolveram em uma briga (uma luta } \\
\text { física). }\end{array}$ & $\begin{array}{l}0.222 \\
(0.002)\end{array}$ & $\begin{array}{l}0.234 \\
(0.003)\end{array}$ \\
\hline Bullying & $\begin{array}{l}\text { Proporção de alunos na escola que responderam que, nos } \\
\text { últimos } 30 \text { dias que antecederam a pesquisa, esculacharam, } \\
\text { zombaram, magoaram, intimidaram ou caçoaram de } \\
\text { algum de seus colegas da escola, tanto que este se sentiu } \\
\text { magoado, aborrecido, ofendido ou humilhado. }\end{array}$ & $\begin{array}{c}0.184 \\
(0.002)\end{array}$ & $\begin{array}{c}0.189 \\
(0.003)\end{array}$ \\
\hline & Variáveis de características dos alunos & & \\
\hline Sexo & Proporção meninos na escola. & $\begin{array}{l}0.471 \\
(0.003)\end{array}$ & $\begin{array}{l}0.475 \\
(0.003)\end{array}$ \\
\hline
\end{tabular}




\section{Variáveis de características dos alunos}

brão $\quad$ Proporção de alunos não brancos na escola.

$0.669 \quad 0.651$

(ancos Proporcaio de alunos nato brancos na escola.

(0.003) (0.003)

Idade Média de idade.

$14.365 \quad 14.369$

(0.005) (0.006)

Trabalha Proporção de crianças que trabalham.

$0.142 \quad 0.145$

(0.002) (0.002)

Carro Proporção alunos na escola que moram com alguém que

$0.461 \quad 0.499$

possui carro.

(0.003) (0.003)

Faculda- Proporção de alunos na escola que a mãe tem faculdade.

de mãe

$0.132 \quad 0.14$

Lar Proporção de alunos na escola que residem apenas com o unipar. pai ou com a mãe.

(0.002) (0.002)

$0.436 \quad 0.441$

(0.003) (0.003)

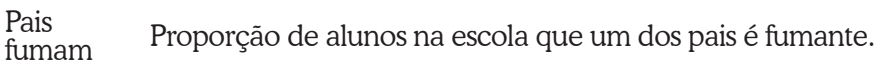

$0.251 \quad 0.254$

Pais não

ajudam

na lição

Proporção de alunos na escola cujos pais nunca ou

raramente verificam se a lição de casa foi feita.

(0.002) (0.003)

Não

almoça

Proporção de alunos na escola que nunca ou raramente

$0.424 \quad 0.44$

com os almoçam ou jantam com seus pais.

(0.003) (0.003)

pais

\section{Variáveis da escola}

$\begin{array}{ll}\text { Tot. } & \text { Escola com até } 200 \text { alunos (base). }\end{array}$

Tot.
Alunos 1 Binária igual a 1 se a escola tem entre 201 e 1.000 alunos.

$0.192 \quad 0.191$

$(0.002) \quad(0.003)$

Tot.

Alunos 2 Binária igual a 1 se a escola tem mais de 1.000 alunos.

$0.664 \quad 0.6$

(0.003) (0.003)

Binária igual a 1 se a escola tem mais de 1.000 alunos.

$0.307 \quad 0.362$

(0.003) (0.003)

Esfera Binária igual a 1 se a escola é federal.

$0.494 \quad 0.236$

Conselho Binária igual a 1 se a escola tem conselho escolar.
Pátio $\quad \begin{aligned} & \text { Binária igual a 1 se o pátio da escola é utilizado para prática } \\ & \text { regular de atividade física com instrutor. }\end{aligned}$

(0.003) (0.003)

$0.903 \quad 0.899$

(0.002) (0.002)

Biblio- Binária igual a 1 se a escola tem biblioteca em condições
teca de uso.

$0.523 \quad 0.441$

(0.003) (0.003)

$0.792 \quad 0.806$

(0.002) (0.003)

Labora- Binária igual a 1 se a escola tem sala ou laboratório de

$0.677 \quad 0.661$

tório informática em condições de uso para os alunos.

(0.003) (0.003) 
conclusão.

\section{Variáveis da escola}

\begin{tabular}{|c|c|c|c|}
\hline $\begin{array}{l}\text { Área de } \\
\text { risco }\end{array}$ & $\begin{array}{l}\text { Binária igual a } 1 \text { se a localidade onde está situada a escola } \\
\text { foi considerada área de risco em termos de violência } \\
\text { (roubos, furtos, assaltos, troca de tiros, consumo de drogas, } \\
\text { homicídios etc.). }\end{array}$ & $\begin{array}{c}0.542 \\
(0.003)\end{array}$ & $\begin{array}{c}0.578 \\
(0.003)\end{array}$ \\
\hline Urbana & Binária igual a 1 se a escola localiza-se na área urbana. & $\begin{array}{c}0.903 \\
(0.002)\end{array}$ & $\begin{array}{c}0.904 \\
(0.002)\end{array}$ \\
\hline Capital & Binária igual a 1 se a escola localiza-se na capital estadual. & $\begin{array}{c}0.446 \\
(0.003)\end{array}$ & $\begin{array}{c}0.46 \\
0.003)\end{array}$ \\
\hline Reg. 0 & Nordeste (base). & & \\
\hline Reg. 1 & Norte. & $\begin{array}{c}0.241 \\
(0.002)\end{array}$ & $\begin{array}{r}0.300 \\
(0.003)\end{array}$ \\
\hline Reg. 2 & Sudeste. & $\begin{array}{c}0.155 \\
(0.002)\end{array}$ & $\begin{array}{c}0.17 \\
(0.002)\end{array}$ \\
\hline Reg. 3 & Sul. & $\begin{array}{c}0.088 \\
(0.002)\end{array}$ & $\begin{array}{c}0.118 \\
(0.002)\end{array}$ \\
\hline Reg. 4 & Centro-oeste. & $\begin{array}{c}0.128 \\
(0.002) \\
\end{array}$ & $\begin{array}{r}0.155 \\
(0.002) \\
\end{array}$ \\
\hline Obs. & & 1.389 & 1.018 \\
\hline
\end{tabular}

Fonte: IBGE Pense, 2015.

Nota 1: Valores da amostra selecionada para as estimações; Erro Padrão entre parênteses.

O consumo do álcool é mais frequente entre os adolescentes, uma vez que mais da metade declarou já ter tomado pelo menos uma dose, seguido do cigarro, com mais de $19 \%$, e das drogas, com mais de $8 \%$. No caso das variáveis de violência, mais de $18 \%$ dos alunos declararam ter cometido bullying nos últimos 30 dias e mais de $22 \%$ declararam envolvimento em uma briga nos últimos 12 meses.

A Figura 1 apresenta as densidades de probabilidade das variáveis de resultados das escolas que aderiram ao PSE e também das que não aderiram. De modo geral, para quase toda a distribuição, as escolas que aderiram ao PSE possuem menor proporção de alunos que consumiram drogas, álcool e cigarros ou que se envolveram em uma briga em relação àquelas que não aderiram ao programa, uma vez que a distribuição das escolas com PSE está à direita em relação às demais. No caso da prática de bullying, essa relação é menos evidente. 
Figura 1 - Distribuição das variáveis de resultados do PSE

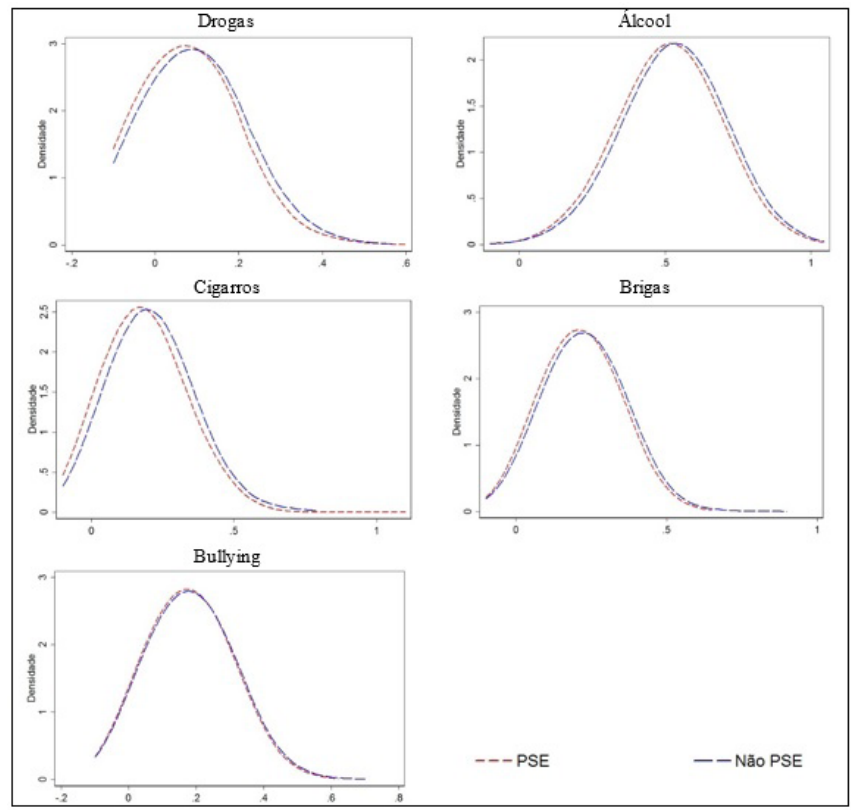

Fonte: Pense (2015)

Conforme descrito no item 2.2 deste artigo, qualquer escola está apta para aderir ao programa, de modo que a participação não é aleatória. Dessa forma, são necessárias variáveis de características observáveis das escolas para construir o contrafatual comparável com as escolas no grupo tratamento. Essas variáveis também foram descritas na Tabela 1 e compreendem as características médias dos alunos e as características da escola.

As variáveis das características médias dos alunos buscam captar aspectos da composição do grupo de escolares. As variáveis sexo, cor e idade referem-se às características pessoais dos alunos. Se os alunos trabalham para complementar a renda da família, a variável trabalha pode ser uma medida da vulnerabilidade econômica dos alunos na escola. Da mesma forma, a proporção de alunos na escola que moram com alguém que possui carro pode ser uma medida para a renda da família. As variáveis faculdade da mãe e lar uniparetal buscam identificar a estrutura da família, de modo que a primeira também pode estar relacionada à uma maior renda familiar. Já os pais fumantes seriam uma medida do exemplo de conduta para uma atividade prejudicial à saúde. As variáveis que identificam os alunos que não recebem ajuda para fazer a lição de casa ou que não almoçam com os pais são medidas para o envolvimento dos pais com as atividades dos filhos. 
As variáveis das características da escola buscam captar aspectos de infraestrutura, organização e localização das escolas. A variável esfera identifica as escolas federais, e a variável total de alunos identifica o tamanho da escola. Já a presença de conselho escolar pode ser uma medida da estrutura administrativa da instituição de ensino. As variáveis pátio, biblioteca e laboratório caracterizam a infraestrutura. A área de risco caracteriza a localidade da escola em termos de violência e as variáveis capital, urbana e localização são características gerais de localização.

\section{Resultados}

A Tabela 2 apresenta os resultados do modelo propensity score logístico que estima a probabilidade de participação das escolas no PSE. Foram incluídas no modelo todas as características observadas que podem afetar tanto o tratamento (PSE) quanto os resultados analisados, que são a prevenção ao uso do álcool, cigarro e outras drogas e a promoção da cultura da paz e prevenção das violências (brigas e bullying). A amostra é composta por 2.407 escolas públicas.

Tabela 2 - Resultados do modelo logit que estima a probabilidade de participação no PSE

\begin{tabular}{lcccc}
\hline Variável & Coeficiente & $\begin{array}{c}\text { Erro } \\
\text { padrão }\end{array}$ & $\begin{array}{c}\text { Efeito } \\
\text { marginal }\end{array}$ & Erro padrão \\
\hline Sexo & $-0,424$ & 0,396 & $-0,103$ & 0,096 \\
Não brancos & 0,300 & 0,360 & 0,073 & 0,087 \\
Idade & $-0,154$ & 0,118 & $-0,037$ & 0,029 \\
Trabalha & 0,935 & 0,720 & 0,227 & 0,186 \\
Carro & $-0,142$ & 0,311 & $-0,034$ & 0,075 \\
Faculdade mãe & 0,255 & 0,464 & 0,062 & 0,112 \\
Lar unipar. & $-0,307$ & 0,387 & $-0,074$ & 0,094 \\
Pais fumam & 0,518 & 0,405 & 0,126 & 0,098 \\
Pais não ajudam na lição & $-0,216$ & 0,387 & $-0,052$ & 0,094 \\
Não almoça com os pais & $-0,079$ & 0,515 & $-0,019$ & 0,125 \\
Tot. Alunos 1 & $0,451^{* *}$ & 0,188 & 0,110 & 0,046 \\
Tot. Alunos 2 & 0,346 & 0,214 & 0,082 & 0,050 \\
Esfera & $1,034^{* * *}$ & 0,101 & 0,242 & 0,022 \\
Conselho & 0,025 & 0,149 & 0,006 & 0,036 \\
Pátio & $0,316^{* * *}$ & 0,089 & 0,076 & 0,022 \\
Biblioteca & 0,084 & 0,115 & 0,020 & 0,028 \\
Laboratório & $0,216^{* * *}$ & 0,100 & 0,053 & 0,025 \\
\hline & & & & $00 n$ tinua... \\
& & & &
\end{tabular}


conclusão.

\begin{tabular}{lcccc}
\hline Variável & Coeficiente & $\begin{array}{c}\text { Erro } \\
\text { padrão }\end{array}$ & $\begin{array}{c}\text { Efeito } \\
\text { marginal }\end{array}$ & Erro padrão \\
\hline Área de risco & $-0,031$ & 0,097 & $-0,008$ & 0,023 \\
Urbana & $0,436^{* * *}$ & 0,165 & 0,107 & 0,041 \\
Capital & 0,064 & 0,108 & 0,015 & 0,026 \\
Reg. 1 - Norte & $-0,514^{* * *}$ & 0,135 & $-0,126$ & 0,033 \\
Reg. 2 - Sudeste & $-0,906^{* * *}$ & 0,149 & $-0,223$ & 0,036 \\
Reg. 3 - Sul & $-0,639^{* * *}$ & 0,218 & $-0,158$ & 0,054 \\
Reg. 4 - Centro-oeste & $-0,577^{* * *}$ & 0,171 & $-0,143$ & 0,042 \\
Log likelihood & $-1527,5418$ & & & \\
\hline
\end{tabular}

Fonte: Elaboração própria a partir dos microdados da Pense 2015 (IBGE).

Nota: ***, **, * denotam significância ao nível de 1\%, 5\% e 10\% respectivamente.

As variáveis de características médias dos alunos e de suas famílias não foram significativas, porém muitos estudos apresentaram evidências de que essas características são importantes determinantes do consumo de substâncias ilícitas e de práticas agressivas dos jovens (CASE; KATZ, 1991; LEVITT; LOCHNER, 2001; GAVIRIA; RAPHAEL, 2001; BECKER; KASSOUF, 2016; BECKER, 2017a).

As escolas federais têm $24 \%$ maior probabilidade de aderir ao PSE, assim como as escolas que possuem entre 201 e 1.000 alunos têm 11\% maior probabilidade em relação às escolas que possuem até 200 alunos. As escolas que possuem laboratório e as que utilizam o pátio para promover atividades físicas para os alunos apresentam, respectivamente, 5\% e 8\% maior probabilidade de participar do programa, assim como as escolas na área urbana e na região Nordeste.

A Tabela 3 apresenta os resultados do efeito médio do tratamento (ATT), obtido por meio do propensity score matching. Os resultados do teste de balanceamento para o matching estão no Apêndice A. A possiblidade de haver variáveis omitidas que influenciam o resultado e o fato de a adesão ao PSE não ser aleatória - uma vez que primeiramente é uma decisão do município participar do programa, para só a partir disso as escolas poderem aderir ao programa - podem levar a estimativas viesadas do efeito do tratamento.

Tabela 3 - Estimação do efeito de tratamento médio

\begin{tabular}{llllll}
\hline & Droga & Álcool & Cigarro & Briga & Bullying \\
\hline ATT & $-0,009^{* * *}$ & $-0,010$ & $-0,011^{*}$ & $-0,012^{* *}$ & $-0,001$ \\
& $(0,004)$ & $(0,007)$ & $(0,005)$ & $(0,005)$ & $(0,004)$ \\
$\mathrm{t}$ & $-2,30$ & $-1,46$ & $-1,93$ & $-2,50$ & $-0,30$ \\
\hline
\end{tabular}

continua... 
conclusão.

\begin{tabular}{llllll}
\hline & Droga & Álcool & Cigarro & Briga & Bullying \\
\hline ATT $_{\text {GMM }}$ & $-0,005^{*}$ & $-0,004$ & $-0,009 * *$ & $-0,007 *$ & $-0,0003$ \\
\hline & $(0,003)$ & $(0,005)$ & $(0,004)$ & $(0,004)$ & $(0,004)$ \\
$\mathrm{t}$ & $-1,92$ & $-0,87$ & $-2,15$ & $-1,85$ & $-0,08$ \\
\hline Hansen J $\left(\chi^{2}\right)$ & 27,204 & 34,281 & 31,078 & 25,235 & 32,576 \\
p-valor & 0,2474 & 0,0612 & 0,1864 & 0,5057 & 0,1747 \\
\hline
\end{tabular}

Fonte: Elaboração própria a partir dos microdados da Pense 2015 (IBGE).

Nota: ***, ** * denotam significância ao nível de 1\%, 5\% e 10\% respectivamente; os desvios padrões robustos estão apresentados entre parênteses, com bootstrap 1000 replicações.

Dessa forma, a Tabela 3 também apresenta o efeito médio do tratamento estimado pelo método generalizado de momentos (ATT ${ }_{\mathrm{GMM}}$ ) a partir do método de Lewbel (2012), que consiste em explorar a heterocedasticidade dos erros no primeiro estágio da regressão para gerar internamente instrumentos que possibilitem a identificação de um sistema de equações.

Os resultados do teste de Breusch e Pagan confirmam heterocedasticidade dos erros $(\chi 2=14,13)$, condição para a validade do método. Os resultados do teste de sobreidentificação das restrições $\mathrm{J}$ de Hansen estão nas duas últimas linhas da Tabela 3. A falha em rejeitar a hipótese nula foi possível em todas as equações, com exceção apenas da equação para o uso do álcool, o que é uma evidência de que, em geral, os instrumentos são válidos. O teste de Kleibergen-Paap Wald permitiu rejeitar a $10 \%$ a hipótese nula de que os instrumentos são fracos, sugerindo que os instrumentos internos gerados são relevantes para o tratamento $(F=1,73)$.

Para o consumo de drogas e cigarros ou envolvimento em brigas, os resultados foram significativos nas estimações por ambos os métodos, o que é uma evidência de que o possível viés causado pelas não observáveis não inviabiliza os coeficientes estimados. No entanto, a magnitude dos coeficientes estimados através do método de Lewbel (2012) foi um pouco menor em relação aos resultados do método convencional, o que pode decorrer de uma possível superestimação do efeito médio quando não são considerados os instrumentos para a participação no PSE.

De maneira geral, os resultados são uma evidência de que a adesão da escola ao PSE reduz a proporção de alunos que declararam ter consumido tais substâncias em aproximadamente 0,01 . Para a prevenção do consumo do álcool, não foram observados resultados significativos, o que pode estar relacionado ao fato de o consumo dessa substância ser socialmente mais aceitável. Ao contrário das drogas e do cigarro, existem propagandas e anúncios publicitários que incentivam o consumo do álcool, o que pode reduzir o poder das ações de conscientização e prevenção do PSE. 
O PSE reduz a proporção de alunos na escola que se envolveram em brigas, porém para a prática de bullying não foram observados resultados significativos. Dessa forma, o efeito do PSE na promoção da cultura da paz e prevenção das violências é observado apenas na forma da violência física. Embora o conceito do que é bullying e dos malefícios dessa prática já sejam bastante discutidos entre os educadores, a linha entre o que é considerado bullying ou uma "brincadeira" entre os alunos é bastante sutil, o que pode tornar mais complexa a definição das ações das políticas de prevenção dessa forma de violência.

Espelage, Low, Polanin e Brown (2013), que avaliam o efeito do Second step: student success through prevention (SS-SSTP) nas escolas americanas, também observaram resultados significativos apenas para a agressão física, de modo que, para agressão verbal, vitimização entre pares, provocações homofóbicas e violência sexual, não foram observados resultados do programa. Já a revisão de literatura de Ttofi e Farrington (2011) aponta que muitos programas realizados nas escolas são capazes de diminuir o bullying, porém ressaltam que os mais eficientes são aqueles que incluem reuniões com os pais e métodos disciplinares rigorosos.

A Figura 2 apresenta os resultados do efeito de tratamento quantílico para cada uma das cinco variáveis de resultados, bem como o intervalo de confiança de $95 \%$, representado pelas linhas cinzas, e permite visualizar como os efeitos do PSE se alteram ao longo da distribuição desses resultados nos quantis 0,1 a 0,9. Os coeficientes estimados estão no Apêndice $B$.

Figura 2 - Efeito do tratamento quantílico

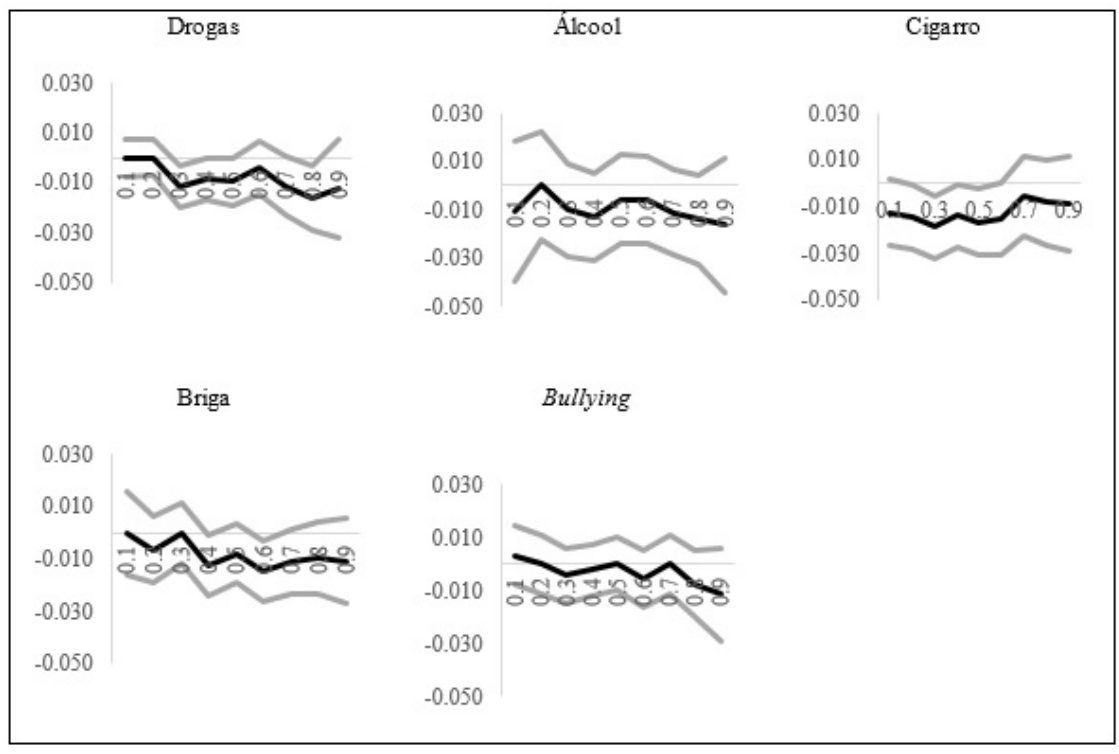

Fonte: Elaboração própria a partir dos microdados da Pense 2015 (IBGE). 
Para o consumo de drogas, os resultados foram significativos entre os quantis 0,3 e 0,8, variando entre -0,010 e -0,016, com exceção do quantil 0,6, que não foi significativo. No caso do consumo de cigarros, os resultados significativos são observados do nível inferior ao nível médio da distribuição, quantis 0,1 a 0,5 , variando entre -0,013 e -0,019. Já para o envolvimento em brigas, os resultados foram significativos apenas nos quantis $0,4,0,6$ e 0,7, variando entre -0,011 e -0,015.

Não foram observados coeficientes significativos no nível superior das distribuições, quantil 0,9, o que pode ser uma evidência de que o PSE não consegue produzir resultados significativos em escolas onde muitos alunos consomem substâncias ilícitas ou praticam violência. Da mesma forma, Caria, Faggiano, Bellocco e Galanti (2011) avaliam o efeito de um programa de prevenção do abuso de substâncias psicotrópicas nas escolas de sete países europeus sobre o consumo de álcool e não observaram resultados significativos entre os estudantes que bebiam com frequência.

$O$ fato de os efeitos observados do PSE serem reduzidos, em torno de 0,01 para o consumo de drogas, cigarros e envolvimento em brigas, ou não significativos para o consumo do álcool e prática de bullying, pode desencadear questionamentos a respeito da validade da intervenção nas linhas de ação referentes à prevenção ao uso do álcool, tabaco e outras drogas e a promoção da cultura da paz e prevenção das violências.

Embora o programa estabeleça o acompanhamento dos educandos com danos decorrentes do uso de substâncias psicotrópicas, as perguntas avaliadas neste estudo são pregressas, ou seja, se alguma vez na vida o estudante declarou ter consumido tais substâncias. Assim, o que está sendo avaliado no estudo é o efeito do programa na prevenção e, nesse sentido, é de se esperar que leve certo tempo até que se possa observar efeitos mais expressivos.

As ações preventivas previstas no programa para atuar nas duas linhas avaliadas neste estudo são atividades educativas e participativas, como rodas de conversa, teatro, dinâmicas, narrativas e outras. Os primeiros manuais disponibilizados pelo governo com sugestões para essas atividades são do ano de 2015 (BRASIL, 2015a, 2015b).

Além disso, a demora no repasse dos recursos para a execução das ações previstas no programa podem prejudicar a efetivação mais imediata dos resultados. Embora o PSE tenha sido instituído em dezembro de 2007, a primeira portaria que habilitou os municípios a receber o repasse financeiro para o PSE é de dezembro de 2011. Para o ano de 2015, no qual se referem os dados deste estudo, há portarias que habilitam os municípios a receber os recursos do PSE de 2013 e de 2014 (Portaria $\mathrm{n}^{\circ} 1.260$, de 27 de agosto de 2015, e Portaria $\mathrm{n}^{\circ} 1.067$, de 23 de julho de 2015).

Diante disso, embora numericamente ainda pequenos, os efeitos significativos do PSE observados neste estudo são evidências de que o programa tem potencial como política de prevenção para a redução do consumo de substâncias psicotrópicas e da violência no longo prazo, atuando sobre o comportamento de crianças e adolescentes, ainda em processo de formação da conduta moral e social. 
Vale lembrar que vários estudos apresentam evidências de que o consumo de substâncias psicotrópicas e a violência reduzem a proficiência do aluno e podem desencadear problemas de saúde, além de gerar mais violência (GAMA; SCORZAFAVE, 2013; TEIXEIRA; KASSOUF, 2015; FIRPO; JALES; PINTO, 2015; ALMEIDA; ARAÚJO JÚNIOR, 2016; MONTEIRO; ROCHA, 2017; BECKER, 2017a). Essas situações representam custos para a sociedade, pois, além de comprometerem a formação do capital humano dos jovens e, consequentemente, da capacidade produtiva futura, aumentam a demanda por segurança pública e pelo sistema de saúde (BECKER; KASSOUF, 2017; BECKER, 2017b).

\subsection{Resultados das Bounds para o Efeito de Tratamento}

A Tabela 4 apresenta os resultados das estimações das bounds para o efeito de tratamento, conforme o método proposto por Oster (2016). Dessa forma, estimou-se o valor do coeficiente de proporcionalidade ( $\delta$ ) que seria necessário para fazer a estimativa do efeito de tratamento ir para zero $(\beta=0)$ e também o efeito do tratamento $(\beta)$ correspondente à solução que minimiza a diferença quadrática para o efeito estimado do tratamento na regressão controlada e que não altera a direção do viés $(\delta$ =1). Para cada variável de resultado, foram considerados quatro valores de Rmax.

Tabela 4 - Bounds para o efeito do tratamento

\begin{tabular}{lllll}
\hline$R_{\text {max }}$ & 0,7 & 0,8 & 0,9 & 1,0 \\
\hline Droga & & & \\
\hline$\delta$ para $\beta=0$ & 1,916 & 1,554 & 1,306 & 1,127 \\
$\beta$ para $\delta=1$ & $-0,005$ & $-0,004$ & $-0,002$ & $-0,001$ \\
\hline Álcool & & & \\
\hline$\delta$ para $\beta=0$ & 0,836 & 0,657 & 0,541 & 0,460 \\
$\beta$ para $\delta=1$ & $-0,001$ & $-0,003$ & $-0,005$ & $-0,008$ \\
\hline Cigarro & & & 1,712 & 1,486 \\
\hline$\delta$ para $\beta=0$ & 2,460 & 2,019 & $-0,008$ & $-0,006$ \\
$\beta$ para $\delta=1$ & $-0,012$ & $-0,010$ & & 1,796 \\
\hline Briga & & & 2,044 & $-0,005$ \\
\hline$\delta$ para $\beta=0$ & 2,826 & 2,372 & $-0,005$ & \\
$\beta$ para $\delta=1$ & $-0,006$ & $-0,006$ & & 0,235 \\
\hline Bullying & & & 0,265 & $-0,094$ \\
\hline$\delta$ para $\beta=0$ & 0,353 & 0,302 & $-0,079$ & \\
$\beta$ para $\delta=1$ & $-0,054$ & $-0,066$ & & \\
\hline
\end{tabular}

Fonte: Elaboração própria a partir dos microdados da Pense 2015 (IBGE). 
Considerando-se o $R_{\max }$ de 0,7 , o efeito das variáveis não observadas no ATT estimado teria de ser 1,92 vezes mais forte do que o efeito das variáveis observáveis para explicar todo o efeito do PSE sobre o consumo de drogas. Em outras palavras, as variáveis não observáveis teriam que ser 1,92 vezes mais importantes na explicação do efeito médio, de modo a fazer com que o coeficiente baseado nas observáveis fosse zero. Aumentando-se o $R_{\max }$ para 1, tal coeficiente ainda é de 1,13. A estimativa do efeito do tratamento considerando-se que a seleção nos controles observáveis é proporcional aos não observáveis $(\delta=1)$ é -0,005 (para $R_{\max }=0,7$ ) e passa para $-0,001$ (para $R_{\text {max }}=1,0$ ).

No caso do efeito do PSE sobre o consumo de cigarro, o efeito das variáveis não observadas no ATT estimado teria de ser 2,46 vezes mais forte do que o efeito das variáveis observáveis, considerando-se o $R_{\max }$ de 0,7 . Para o $R_{\max }$ de 1,0 , esse valor é 1,49 . A estimativa do efeito do tratamento considerando-se $\delta=1$ é $-0,012$ (para $R_{\max }=0,7$ ) e passa para $-0,006$ (para $R_{\max }=1,0$ ). Já para o envolvimento em brigas, os resultados de $\delta$ foram 2,83 e 1,80 e de $\beta$ foram de -0,006 e -0,005, considerando-se, respectivamente, o $R_{\max }$ de 0,7 e de 1,0 .

Esses resultados corroboram as evidências da Tabela 3 de que, no caso do consumo de drogas, cigarros e envolvimento em brigas, o viés causado pelas não observáveis não inviabiliza os coeficientes estimados. No caso do consumo de álcool e bullying, a estimativa de $\delta$ foi menor do que um, o que é um indicativo de que as variáveis mais importantes para explicar o tratamento são não observáveis, de modo que os resultados das estimativas do ATT podem ser viesados.

\section{Considerações Finais}

Este estudo analisou o efeito do PSE em cinco resultados, que são a proporção de alunos na escola que declaram já ter consumido drogas, álcool e/ou cigarros, ter se envolvido em brigas e/ou praticado bullying, com base nas informações da Pense 2015. Para isso, estimou-se o efeito médio do tratamento nos tratados (ATT) e o efeito do tratamento quantílico. O ATT foi estimado pelo método de propensity score matching, que se baseia nas características observáveis dos alunos e das escolas, e também pelo método de Lewbel (2012), que busca controlar o possível viés causado pela influência das características não observáveis sobre o tratamento por meio da construção de instrumentos internamente, a partir da heterocedasticidade do termo do erro.

Para o consumo de drogas e cigarros ou envolvimento em brigas, os coeficientes estimados do ATT foram significativos e semelhantes nas estimações por ambos os métodos, indicando que a proporção de jovens que apresentam tais comportamentos, matriculados nas escolas que aderiram ao PSE, é menor em comparação às demais escolas, em aproximadamente 0,01 . A estimativa das bounds para o efeito 
do tratamento também indicaram que o possível viés causado pelas não observáveis não inviabiliza os coeficientes estimados no caso desses três resultados.

Para o consumo do álcool e prática de bullying, que são mais comuns entre os jovens, não foram observados resultados significativos. O bullying é, muitas vezes, interpretado como uma "brincadeira" entre os jovens, e o consumo do álcool costuma ser socialmente mais aceitável e incentivado por propagandas e anúncios publicitários. Essas situações tornam mais complexa a definição das ações das políticas de prevenção de tais práticas.

Nas regressões quantílicas, o efeito do tratamento não foi significativo no nível superior das distribuições, quantil 0,9, dos cinco resultados analisados, o que pode ser uma evidência de que o PSE não consegue produzir efeito em escolas onde muitos alunos consomem substâncias ilícitas ou praticam violência.

\section{REFERÊNCIAS}

ANGRIST, J. D.; PISCHKE, J. Mostly harmless econometrics: an empiricist's companion. Princeton: Princeton University Press, 2009.

ANDERSON, D. M. In school and out of trouble? The minimum dropout age and juvenile crime. The Review of Economics and Statistics, v. 96, n. 2, p. 318-331, 2014.

ALMEIDA, A. T. C.; ARAÚJO JÚNIOR, I. T. Efeitos da exposição aos fatores de risco comportamentais à saúde sobre o atraso escolar no Brasil. Revista Brasileira de Economia-RBE, v. 70, n. 2, p. 129-169, 2016.

BECKER, K. L. O efeito da interação social entre os jovens nas decisões de consumo de álcool, cigarros e outras drogas ilícitas. Estudos Econômicos, v. 47, n. 1, p. 65-92, 2017a.

BECKER, K. L. Análise do efeito dos gastos públicos na área social sobre o consumo de drogas dos alunos nas escolas das capitais brasileiras. In: ENCONTRO NACIONAL DA ASSOCIAÇÃO BRASILEIRA DE ESTUDOS REGIONAIS E URBANOS, 15., 2017, São Paulo. Anais eletrônicos [...]. São Paulo: FEAUSP: NEREUS, 2017b.

BECKER, K. L. KASSOUF, A. L. Uma análise do efeito dos gastos públicos em educação sobre a criminalidade no Brasil. Economia e Sociedade - UNICAMP, v. 26, p. 215-242, 2017.

BECKER, K. L. KASSOUF, A. L. Violência nas escolas públicas brasileiras: uma análise da relação entre o comportamento agressivo dos alunos e o ambiente escolar. Nova Economia - UFMG, v. 26, p. 653-677, 2016.

BRASIL. Decreto ${ }^{\circ}$ 6.286, de 5 de dezembro de 2007. Institui o Programa Saúde na Escola - PSE, e dá outras providências. Diário Oficial [da] República Federativa do Brasil. Brasília, DF, 5 de dezembro de 2007. 
BRASIL. Secretaria Nacional Antidrogas. VI Levantamento nacional sobre o consumo de drogas psicotrópicas entre estudantes do ensino fundamental e médio das redes pública e privada de ensino nas 27 capitais brasileiras 2010. São Paulo: CEBRID: UNIFESP, 2010.

BRASIL. Ministério da Saúde; Ministério da Educação. Passo a passo PSE programa saúde na escola: tecendo caminhos da intersetorialidade. Brasília: Ministério da Saúde: Ministério da Educação, 2011a.

BRASIL. Portaria n ${ }^{\circ}$ 3.014, de 20 de dezembro de 2011. Habilita Municípios e o Distrito Federal para o recebimento do repasse de recursos financeiros relativos ao Programa Saúde na Escola (PSE). Diário Oficial [da] República Federativa do Brasil, Brasília, DF, 20 de dezembro de $2011 \mathrm{~b}$.

BRASIL. Portaria interministerial $\mathrm{n}^{\circ} 1.413$, de 10 de julho de 2013. Redefine as regras e critérios para adesão ao Programa Saúde na Escola (PSE) por Estados, Distrito Federal e Municípios e dispõe sobre o respectivo incentivo financeiro para custeio de ações. Diário Oficial [da] República Federativa do Brasil, Brasília, DF, 10 de julho de 2013.

BRASIL. Ministério da Saúde; Ministério da Educação. Guia de sugestões de atividades: semana saúde na escola. Brasília: Ministério da Saúde: Ministério da Educação, 2015a.

BRASIL. Ministério da Saúde; Ministério da Educação. Caderno temático: direitos humanos. Brasília: Ministério da Saúde: Ministério da Educação, 2015b.

BRASIL. Portaria $n^{\circ}$ 1.067, de 23 de julho de 2015. Define Municípios com adesão ao Programa Saúde na Escola em 2014 e os habilita ao recebimento de 20\% (vinte por cento) do teto de recursos financeiros pactuados em Termo de Compromisso e dá outras providências. Diário Oficial [da] República Federativa do Brasil, Brasília, DF, 23 de julho de 2015c.

BRASIL. Portaria $\mathrm{n}^{\circ} 1.260$, de 27 de agosto de 2015. Habilita Municípios ao recebimento do repasse de recursos financeiros relativos ao Programa Saúde na Escola 2013. Diário Oficial [da] República Federativa do Brasil, Brasília, DF, 27 de agosto de 2015d.

CARIA, M. P.; FAGGIANO, F.; BELLOCCO, R.; GALANTI, M. R. Effects of a school-based prevention program on European adolescents' patterns of alcohol use. Journal of Adolescent Health, v. 48, p. 182-188, 2011.

CASE, A.; KATZ, L. The company you keep: the effects of family and neighborhood on disadvantaged youths. NBER Working Paper, n. 3705, 1991.

EMRAN, M. S.; HOU, Z. Access to markets and rural poverty: evidence from household consumption in China. The Review of Economics and Statistics, v. 95, n. 2, p. 682-697, 2013.

ESPELAGE, D. J.; LOW, S.; POLANIN, J. R M. A.; BROWN, E. C. The impact of a middle school program to reduce aggression, victimization, and sexual violence. Journal of Adolescent Health, v. 53, n. 2, p. 180-186, 2013.

FAGGIANO, F.; VIGNA-TAGLIANTI, F.; BURKHART, G.; BOHRN, K.; CUOMO, L.; GREGORI, D.; PANELLA, M.; SCATIGNA, M.; SILIQUINI, R.; VARONA, L.; KREEFT, P.; VASSARA, M.; WIBORG, G.; GALANTIL, M. R. The effectiveness of a school-based substance abuse preven- 
tion program: 18-month follow-up of the EU-Dap cluster randomized controlled trial. Drug and Alcohol Dependence, v. 108, n. 1-2, p. 56-64, 2010.

FIRPO, S.; JALES, H.; PINTO, C. Measuring peer effects in the Brazilian school system. Applied Economics, v. 47, n. 32, 2015.

GAMA, V. A.; SCORZAFAVE, L. G. Os efeitos da criminalidade sobre a proficiência escolar no ensino fundamental no município de São Paulo. Pesquisa e Planejamento Econômico PPE, v. 43, n. 3, 2013.

GAVIRIA, A.; RAPHAEL, S. School-based peer effects and juvenile behavior. The Review of Economics and Statistics, v. 83, n. 2, p. 257-268, 2001.

JACOB, B. A. LEFGREN, L. Are idle hands the devil's workshop? Incapacitation, concentration, and juvenile crime. American Economic Review, v. 93, n. 5, p. 1560-1577, 2003.

KOENKER, R.; BASSETT, G. Regression quantiles. Econometrica, v. 46, n. 1, p. 33-50, 1978.

LEVITT, S. D.; LOCHNER L. The determinants of juvenile crime. In: GRUBER, J. (ed.). Risky behavior among youths: an economic analysis. Chicago: University of Chicago Press, 2001. p. 327-374.

LEWBEL, A. Using heteroscedasticity to identify and estimate mismeasured and endogenous regressor models. Journal of Business and Economic Statistics, v. 30, p. 67-80, 2012.

MONTEIRO, J. ROCHA, R. Drug battles and school achievement: evidence from Rio de Janeiro's favelas. The Review of Economics and Statistics, v. 99, n 2, p. 213-228, 2017.

OSTER, E. Unobservable selection and coefficient stability: theory and evidence. Working paper, Brown University, 2016.

PENSE: pesquisa nacional de saúde do escolar. Microdados, 2015. Rio de Janeiro: IBGE, 2015.

ROSENBAUM, P.; RUBIN, D. Constructing a control group using multivariate matched sampling methods that incorporate the propensity score. The American Statistician, v. 39, n. 1, p. 33-38, 1985.

TEIXEIRA, E. C.; KASSOUF, A. L. Impacto da violência nas escolas paulistas sobre o desempenho acadêmico dos alunos. Economia Aplicada, v. 19, n. 2, 2015, p. 221-240, 2015.

TIGRE, R.; SAMPAIO, B.; MENEZES, T. The impact of commuting time on youth's school performance. Journal of Regional Science, v. 57, n. 1, 2016.

TTOFI, M. M.; FARRINGTON, D. P. Effectiveness of school-based programs to reduce bullying: a systematic and meta-analytic review. Journal of Experimental Criminology, v. 7, n. 1, p. 27-56, 2011. 


\section{Apêndice A - Resultados do Teste de Balanceamento para o Matching}

\begin{tabular}{|c|c|c|c|c|}
\hline Variável & Tratamento & Controle & $t$ & p-valor \\
\hline Sexo & 0,479 & 0,478 & 0,21 & 0,836 \\
\hline Não brancos & 0,673 & 0,670 & 0,44 & 0,661 \\
\hline Idade & 14,424 & 14,435 & $-0,73$ & 0,466 \\
\hline Trabalha & 0,140 & 0,142 & $-0,44$ & 0,656 \\
\hline Carro & 0,675 & 0,681 & $-0,68$ & 0,5 \\
\hline Faculdade mãe & 0,117 & 0,120 & $-0,68$ & 0,496 \\
\hline Lar unipar. & 0,430 & 0,440 & $-1,65$ & 0,099 \\
\hline Pais fumam & 0,269 & 0,268 & 0,1 & 0,92 \\
\hline Pais não ajudam na lição & 0,419 & 0,422 & $-0,56$ & 0,576 \\
\hline Não almoça com os pais & 0,198 & 0,201 & $-0,8$ & 0,426 \\
\hline Tot. Alunos 1 & 0,735 & 0,736 & $-0,06$ & 0,951 \\
\hline Tot. Alunos 2 & 0,204 & 0,202 & 0,13 & 0,9 \\
\hline Esfera & 0,525 & 0,513 & 0,68 & 0,498 \\
\hline Conselho & 0,899 & 0,892 & 0,57 & 0,566 \\
\hline Pátio & 0,516 & 0,512 & 0,21 & 0,837 \\
\hline Biblioteca & 0,748 & 0,769 & $-1,28$ & 0,199 \\
\hline Laboratório & 0,634 & 0,631 & 0,18 & 0,856 \\
\hline Área de risco & 0,513 & 0,517 & $-0,25$ & 0,805 \\
\hline Urbana & 0,842 & 0,847 & $-0,37$ & 0,709 \\
\hline Capital & 0,384 & 0,403 & $-1,01$ & 0,312 \\
\hline Reg. 1 - Norte & 0,228 & 0,241 & $-0,79$ & 0,43 \\
\hline Reg. 2 - Sudeste & 0,131 & 0,116 & 1,19 & 0,232 \\
\hline Reg. 3 - Sul & 0,100 & 0,109 & $-0,8$ & 0,426 \\
\hline Reg. 4 - Centro-oeste & 0,121 & 0,126 & $-0,4$ & 0,692 \\
\hline
\end{tabular}

Fonte: Elaboração própria a partir dos microdados da Pense, 2015.

\section{Apêndice B - Efeito do Tratamento Quantilico}

\begin{tabular}{llllll}
\hline Quantil & Droga & Álcool & Cigarro & Briga & Bullying \\
\hline \multirow{2}{*}{0,1} & 0,000 & $-0,011$ & $-0,013^{*}$ & 0,000 & 0,003 \\
& $(0,004)$ & $(0,015)$ & $(0,007)$ & $(0,008)$ & $(0,006)$ \\
\multirow{2}{*}{0,2} & 0,000 & 0,000 & $-0,014^{* * *}$ & $-0,006$ & 0,000 \\
& $(0,004)$ & $(0,012)$ & $(0,007)$ & $(0,007)$ & $(0,006)$ \\
\hline
\end{tabular}


conclusão.

\begin{tabular}{llllll}
\hline Quantil & Droga & Álcool & Cigarro & Briga & Bullying \\
\hline \multirow{2}{*}{0,3} & $-0,011^{* * *}$ & $-0,01$ & $-0,019^{* * *}$ & 0,000 & $-0,004$ \\
& $(0,004)$ & $(0,01)$ & $(0,007)$ & $(0,006)$ & $(0,005)$ \\
0,4 & $-0,009^{*}$ & $-0,013$ & $-0,014^{* * *}$ & $-0,013^{* *}$ & $-0,002$ \\
& $(0,004)$ & $(0,009)$ & $(0,007)$ & $(0,006)$ & $(0,005)$ \\
0,5 & $-0,01^{* *}$ & $-0,006$ & $-0,017^{* *}$ & $-0,008$ & 0,000 \\
& $(0,005)$ & $(0,009)$ & $(0,007)$ & $(0,006)$ & $(0,005)$ \\
0,6 & $-0,004$ & $-0,006$ & $-0,015$ & $-0,015^{* *}$ & $-0,006$ \\
& $(0,005)$ & $(0,009)$ & $(0,008)$ & $(0,006)$ & $(0,005)$ \\
0,7 & $-0,011^{*}$ & $-0,011$ & $-0,006$ & $-0,011^{*}$ & 0,000 \\
& $(0,006)$ & $(0,009)$ & $(0,009)$ & $(0,006)$ & $(0,006)$ \\
0,8 & $-0,016^{* *}$ & $-0,014$ & $-0,008$ & $-0,01$ & $-0,008$ \\
& $(0,007)$ & $(0,01)$ & $(0,009)$ & $(0,007)$ & $(0,006)$ \\
0,9 & $-0,013$ & $-0,016$ & $-0,009$ & $-0,011$ & $-0,011$ \\
& $(0,01)$ & $(0,014)$ & $(0,01)$ & $(0,008)$ & $(0,009)$ \\
\hline
\end{tabular}

Fonte: Elaboração própria a partir dos microdados do Pense, 2015.

Nota: ***, **, * denotam significância ao nível de 1\%, 5\% e 10\% respectivamente; desvios padrões entre parênteses.

Recebido em: 20/02/2018. Aceito em: 06/09/2018.

\section{(cc) BY}

144 Análise Econômica, Porto Alegre, v. 38, n. 76, p. 121-144, jun. 2020. 